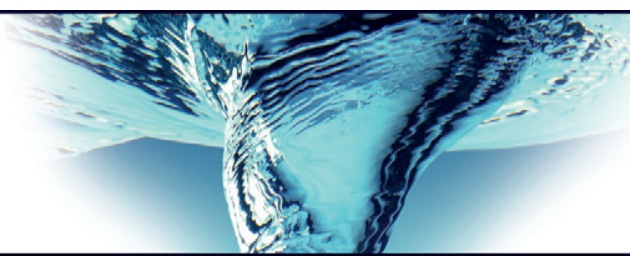

\title{
Turbulent drag reduction by polymer additives in parallel-shear flows
}

\author{
Bayode E. Owolabi ${ }^{1}$, David J. C. Dennis ${ }^{1}$ and Robert J. Poole ${ }^{1, \dagger}$ \\ ${ }^{1}$ School of Engineering, University of Liverpool, Liverpool L69 3GH, UK \\ (Received 16 April 2017; revised 23 June 2017; accepted 1 August 2017; \\ first published online 24 August 2017)
}

In this study, we experimentally investigate the turbulent drag-reduction (DR) mechanism in flow through ducts of circular, rectangular and square cross-sections using two grades of polyacrylamide in aqueous solution having different molecular weights and various semidilute concentrations. Specifically, we explore the relationship between drag reduction and fluid elasticity, purposely exploiting the mechanical degradation of polymer molecules to vary their rheological properties. We also obtain time-resolved velocity data for various DR levels using particle image velocimetry and laser Doppler velocimetry. Elasticity is quantified via relaxation times determined from uniaxial extensional flow using a capillary breakup apparatus. A plot of DR against Weissenberg number (Wi) is found to approximately collapse the data, with the onset of $\mathrm{DR}$ occurring at $W i \approx 0.5$ and the maximum drag-reduction asymptote being approached for $W i \gtrsim 5$. Thus quantitative predictions of DR in a range of shear flows can be made from a single measurable material property of a polymer solution, at least for this particular flexible linear polymer.

Key words: drag reduction, polymers, turbulent flows

\section{Introduction}

It is well known that long-chain flexible polymers of high molecular weight possess excellent drag-reduction capabilities in turbulent flow when added to a Newtonian solvent even at minute concentrations. This phenomenon, discovered by Toms (1948), has prompted a large number of studies to better understand the underlying mechanism, with researchers approaching the problem from theoretical, experimental and numerical perspectives. Periodic overviews of the literature published on the subject can be found in the reviews by Virk (1975), Den Toonder et al. (1995), Graham (2004), Procaccia, Lvov \& Benzi (2008) and White \& Mungal (2008) among others.

\section{$\dagger$ Email address for correspondence: robpoole@liverpool.ac.uk}




\section{B. E. Owolabi, D. J. C. Dennis and R. J. Poole}

One conclusion on which there is a consensus among researchers is that the phenomenon occurs as a result of the dynamical interaction between polymer molecules and turbulence. This interaction begins when their relaxation time becomes comparable to a characteristic time scale of the flow, leading to considerable stretching of the polymer molecules. A detailed analysis by Lumley (1973) shows that this onset criterion is given by the so-called 'coil-stretch' transition, and in a purely extensional flow at strain rate $\dot{\varepsilon}$, this occurs for $W i=\lambda \dot{\varepsilon} \geqslant 1 / 2$, where $W i$ is the Weissenberg number and $\lambda$ is the relaxation time. In a chaotic, turbulent-like flow of mixed shear and extensional flow, Stone \& Graham (2003) have shown that significant stretching occurs when the product of the relaxation time and the largest Lyapunov exponent approaches $1 / 2$. In the current study, $W i$ is approximately estimated via the product of relaxation time and the mean shear rate at the wall $\dot{\gamma}$ such that $W i=\lambda \dot{\gamma}$.

Given the important role played by polymer stretching, an understanding of the extensional rheological properties of polymer solutions has long been felt to be crucial to obtaining a better insight into the drag-reduction mechanism. Until the fairly recent introduction of the commercial Capillary Breakup Extensional Rheometer (CaBER) (Bazilevsky, Entov \& Rozhkov 1990; Rodd et al. 2005), accurate measurements of these properties have been elusive to experimentalists due to the dilute nature of the polymer solutions and the difficulty in creating a truly extensional flow for rheometric measurements. A few studies exist where an attempt has been made to quantitatively relate drag reduction to polymer rheological properties in shear or extension, with little success (Metzner \& Park 1964; Darby \& Chang 1984; James \& Yogachandran 2006). One approach has been to estimate polymer relaxation time from shear rheometric measurements but such experiments are equally challenging for dilute polymers, especially in aqueous solution (Lindner, Vermant \& Bonn 2003; Zell et al. 2010). Another unsuccessful approach to find a correlation was to directly measure the extensional viscosity using opposed-nozzle devices (e.g. Escudier, Presti \& Smith 1999), but the flow fields created by such configurations have been shown to be corrupted by shear and inertia, causing the measurements to differ from the true material property (Dontula et al. 1997). Thus, quantitative predictions of drag-reduction level from a measurable material property have remained elusive.

One of the most important findings on polymer drag reduction in wall-bounded turbulent flows is the existence of a maximum drag-reduction asymptote (MDR) (Virk 1975). Data from gross flow studies plotted in Prandtl-von Kármán coordinates $(1 / \sqrt{f}$ versus $R e^{\star} \sqrt{f}$, where $f$ is the Fanning friction factor and $R e^{\star}$ is the Reynolds number based on duct hydraulic diameter) show that at onset of DR, there is a slope increment from the Newtonian Prandtl-Kármán law that is proportional to polymer concentration. Eventually, an upper bound - MDR - is reached for sufficiently large values of $R e^{\star}$ and/or polymer concentration. The mean velocity profile in wall units for intermediate drag-reducing flows has also been found to lie between the Newtonian log law and MDR asymptote (Virk 1975).

Polymer stretching is believed to bring about a reduction in momentum flux from the bulk to the wall (Procaccia et al. 2008). Experimental observations with polymer additives (Den Toonder et al. 1995) found that wall-normal velocity fluctuations were decreased and the streamwise velocity fluctuations were increased (when these velocities are normalised by the friction velocity, $u_{\tau}$, which is the square root of the ratio of wall shear stress and density, $u_{\tau}=\sqrt{\tau_{w} / \rho}$ ). A major challenge faced by these experimental studies, however, is the mechanical degradation of polymer molecules, leading to poor repeatability and large uncertainties in data, especially in the region between onset and MDR. 


\section{Turbulent drag reduction by polymer additives in parallel-shear flows}

In recent times, numerical simulations of viscoelastic turbulent flows have been conducted using simple models, such as the finite-extensibility nonlinear elastic dumbbell model with the Peterlin approximation (FENE-P), in which a polymer molecule is treated as a pair of spherical beads connected by an elastic spring (Sureshkumar, Beris \& Handler 1997; Housiadas \& Beris 2003; Xi \& Graham 2010). So far the FENE-P model has reproduced some universal features of polymer drag reduction such as the MDR asymptote (see Graham 2014), but it is also known to be unable to correctly predict pressure losses in laminar contraction flows, for example (Purnode \& Crochet 1996), and to overestimate viscoelastic stresses in turbulent flow (Stone \& Graham 2003).

In this study, we experimentally investigate polymer drag reduction in a cylindrical pipe, a rectangular channel and a square duct - each a commonly encountered geometry in the study of wall-bounded turbulence. We carefully characterise our polymer solutions, utilising the effect of mechanical degradation to explore the relationship between drag reduction and fluid elasticity. In so doing, we are able to obtain quantitative predictions for the degree of turbulent drag reduction in a given flow - at least for the polymer studied - as long as CaBER measurements are obtainable.

\section{Experimental arrangements and instrumentation}

The experimental rigs used in this study (figure 1) have similar arrangements to those employed in previous research in our laboratory (Dennis \& Sogaro 2014; Owolabi, Poole \& Dennis 2016; Whalley et al. 2017), with slight modifications. For the pipe flow studies, a $23 \mathrm{~m}$ long pipe consisting of a series of borosilicate glass tubes with internal diameter $(2 R)$ of $100 \mathrm{~mm}$ was employed. The rectangular channel consists of five $1.2 \mathrm{~m}$ long stainless steel sections, each of cross-sectional dimensions $25 \mathrm{~mm} \times 298 \mathrm{~mm}(2 h \times b)$, followed by a $0.25 \mathrm{~m}$ long module fabricated with borosilicate glass sidewalls, to allow for laser Doppler velocimetry (LDV) measurements, and another stainless steel section of length $1.2 \mathrm{~m}$, bringing the total length to $7.45 \mathrm{~m}$. The square duct has a working section consisting of nine stainless steel modules, each having a length of $1.2 \mathrm{~m}$ and cross-sectional dimensions $80 \mathrm{~mm} \times 80 \mathrm{~mm}(2 h \times 2 h)$. A transparent section, $150 \mathrm{~mm}$ in length and constructed from Perspex, is introduced between the eighth and ninth modules to provide optical access for LDV measurements at a distance of about $240 \mathrm{~h}$ from the inlet.

A cylindrical plenum chamber is introduced at the inlet of the pipe to reduce the degree of swirl and ensure uniform flow. Transition sections designed to vary in crosssection from circular to rectangular (or square) or vice versa are also introduced at the inlet and outlet of the rectangular and square channels to ensure smoothly varying flow.

In all three experimental rigs, the working fluid was recirculated using progressive cavity pumps, and pulsation dampers installed immediately after the pumps served to remove possible disturbances in the flow. Mass flow rate, density and temperature were measured using Coriolis mass flow meters (a platinum resistance thermometer was used for temperature measurements in the rectangular channel), while pressuredrop measurements were obtained using a Validyne differential pressure transducer (DP 15-26) over lengths of $165 R, 452 h$ and $215 h$ for the pipe, rectangular channel and square duct respectively.

A Dantec Dynamics stereoscopic particle image velocimetry (SPIV) system was used to obtain the three-component velocity field in the pipe over its entire crosssection. The measurements were taken at a distance of $440 R$ from the inlet. The SPIV 


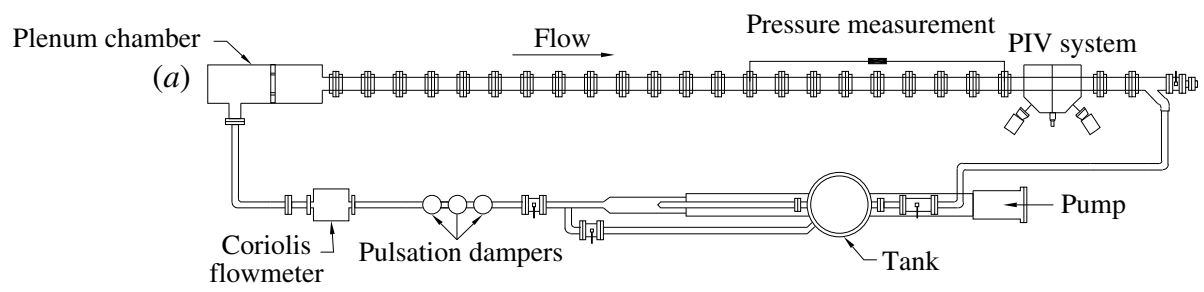

(b)

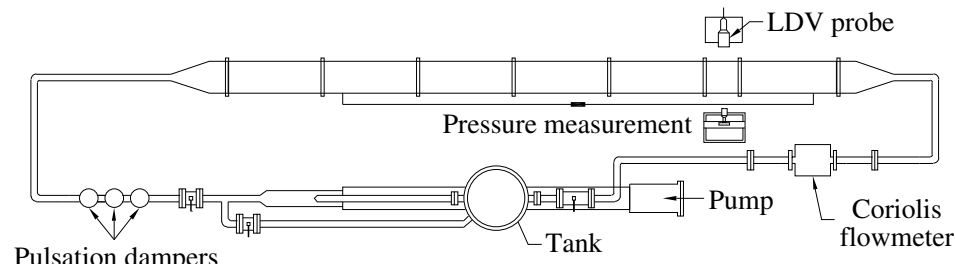

(c)

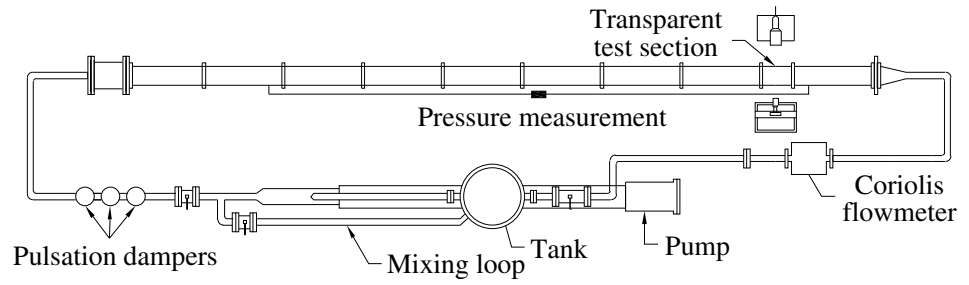

FIGURE 1. Experimental arrangements (not to scale) of the three parallel-shear flows:

(a) pipe, (b) channel and $(c)$ square duct. Flow is clockwise in all three rigs.

system consists of a dual-cavity Nd:YAG Lee laser of wavelength $532 \mathrm{~nm}$ and two high-speed Phantom Miro 110 cameras positioned on opposite sides of the laser light sheet. The pipe measurement section is enclosed in a water-filled prism to provide undistorted optical access for the cameras. The flow was seeded with $10 \mu \mathrm{m}$ silvercoated hollow spheres and velocity data were obtained at sampling rates of up to $619 \mathrm{~Hz}$.

Velocity measurements in the rectangular and square channels were taken using a Dantec Dynamics LDV system operated in forward-scatter mode. This system consisted of a $60 \times 10$ probe, $55 \times 12$ beam expander and an argon-ion laser source that supplied light of wavelength $515.5 \mathrm{~nm}$. The front lens of the laser probe had a focal length of $160 \mathrm{~mm}$ and a beam separation distance of $51.5 \mathrm{~mm}$, resulting in a measuring volume of diameter $24 \mu \mathrm{m}$ and length $150 \mu \mathrm{m}$ in air. With this configuration, the typical data rates were around $100 \mathrm{~Hz}$.

\section{Preparation and rheological characterisation of the working fluid}

Polyacrylamide, a flexible polymer, is known to be a good drag-reducing agent, with skin friction reduction of up to about $75 \%$ recorded in the literature (Escudier, Nickson \& Poole 2009). Two grades of polyacrylamide solutions having different molecular weights with concentrations ranging from 150 to 350 parts per million (p.p.m.) were studied: FloPAM AN934SH ('PAA') and Separan AP273E ('Separan') both supplied by Floreger. Intrinsic viscosity measurements $[\eta]$ for each grade of polymer $\left([\eta]=4400 \mathrm{ml} \mathrm{g}^{-1}\right.$ for PAA and $3400 \mathrm{ml} \mathrm{g}^{-1}$ for Separan) suggest critical overlap concentrations $\left(c^{*}\right)$ of 225 p.p.m. and 300 p.p.m. for PAA and Separan respectively. Thus all concentrations are of the order of $c^{*}$ and are therefore best 


\section{Turbulent drag reduction by polymer additives in parallel-shear flows}

considered semidilute rather than truly dilute (Clasen et al. 2006). (NB. Although there must exist some polydispersity within these polymers, it was not possible to measure their molecular weights using gel phase chromatography (GPC) due to the occurrence of viscous fingering in the GPC column.) Both grades of polyacrylamide, however, do degrade under high shear, hence great care was taken in their preparation. First, stock solutions with concentrations ranging from 1200 to 1500 p.p.m. were manually prepared outside the rigs and left for a period of 24 hours to homogenise before being diluted to the desired concentrations in situ by mixing with water at very low pump speeds. For every run in the drag-reduction studies, the rheological properties of the polymer solutions were closely monitored. Fluid samples were taken at different pumping times for viscosity and relaxation time measurements using an Anton Paar MCR302 controlled-stress torsional rheometer and a CaBER respectively, in conjunction with pressure-drop measurements to estimate the level of drag reduction.

The CaBER comprises two circular stainless steel plattens with a diameter of $4 \mathrm{~mm}$ and an initial separation of $\approx 2 \mathrm{~mm}$. A small sample of each solution was loaded between the plattens using a syringe (without a needle to minimise the shear) to form a cylindrical sample. A rapid axial step strain was imposed $(\approx 50 \mathrm{~ms})$ until a final height $(\approx 9 \mathrm{~mm})$ was reached and an unstable filament formed. Subsequently, the sample filament breaks up under the combined action of capillary and extensional viscoelastic forces. The diameter of the filament was observed as a function of time using the equipment's laser micrometer (resolution $10 \mu \mathrm{m}$ ). Although the data on filament diameter can be postprocessed into an (apparent) extensional viscosity, the standard method (Rodd et al. 2005) to quantify extensional effects is via an exponential fit to the filament diameter as a function of time in the elasto-capillary regime to determine a characteristic relaxation time (more correctly a characteristic time for extensional stress growth).

Example data sets of shear viscosity and the variation of relaxation time with pumping time are shown in figure 2. Polymer degradation can be observed to bring about a large reduction in the zero-shear viscosity, the sharpest drop occurring in the first 45 minutes of pumping (as shown in figure $2 a$ ). Power-law fits to the shear viscosity curves (inset of figure $2 a$ ) also show a decrease in the amount of shear thinning during this time frame. At longer times, the rate of polymer degradation slows down and shear viscosity becomes roughly constant. The relaxation times measured by CaBER $\left(\lambda_{c}\right)$ can be observed to follow a similar trend (see figure $2 b$ ), dropping from relatively large values at the start of pumping before asymptoting to a value of about $4 \mathrm{~ms}$ at long times (close to the minimum resolution of the standard CaBER technique). From the $\log -\log$ plot (inset of figure $2 b$ ), it can be observed that $\lambda_{c}$ scales roughly as $t^{-0.5}$, where $t$ is the pumping time in seconds.

\section{Mean streamwise velocity measurements}

Figure 3 shows the velocity profiles in the pipe, rectangular channel and square duct at different levels of drag reduction. In this study, $D R$ is defined as

$$
\% D R=\frac{\Delta P_{N}-\Delta P_{V}}{\Delta P_{N}} \times\left. 100\right|_{\dot{m}},
$$

where $\Delta P_{N}$ and $\Delta P_{V}$ are the pressure drops in the Newtonian fluid and polymer (viscoelastic) solution respectively, measured at the same mass flow rate, $\dot{m}$. 

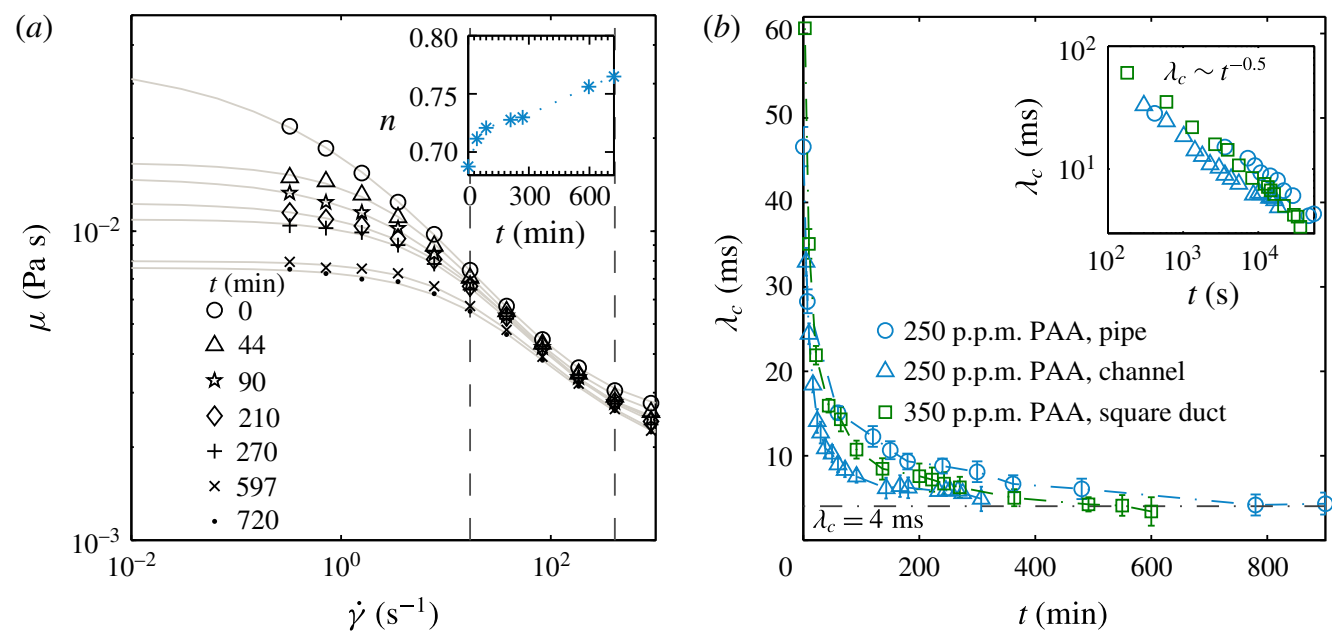

FIGURE 2. Variation of the rheological properties of polymer solutions with pumping time t. (a) Example shear viscosity curves for PAA (concentration of 250 p.p.m. in the square duct) at various stages of mechanical degradation: a power-law model $\left(\mu=k \dot{\gamma}^{n-1}\right)$ has been fitted in the range shown by the two vertical dashed lines and the inset shows the change in power-law index, $n$, with pumping time $t$. (b) Typical relationships between CaBER-measured relaxation time and pumping time, showing rapid degradation, with an inset of the same data on $\log -\log$ axes highlighting $\lambda_{c} \approx t^{-0.5}$.

The time-resolved velocity data were obtained using LDV in the rectangular and square channels and by SPIV in the pipe. With SPIV, it was possible to monitor the changes in the mean velocity profile with decreasing drag-reduction level in a single experiment, since the technique provides velocity information in the entire cross-section of the pipe within a reasonable time frame over which degradation is minimal. With LDV (a pointwise measurement technique), however, useful velocity data could only be obtained after long pumping times when the polymer rheological properties were not changing much and $\% D R$ was roughly constant.

From the plots shown in figure 3, a thickened buffer layer can be clearly seen, extending across the entire cross-section at MDR (72\% DR in the pipe, see figure $3 a$ ). The Weissenberg number for all the polymer data was greater than 1 , indicating the importance of polymer stretching. In the pipe, the profile of $U^{+}=U / u_{\tau}$ lies slightly above Virk's MDR line, $U^{+}=11.7 \ln \left(y^{+}\right)-17$, for $y^{+}>80\left(y^{+}\right.$is the distance from the wall normalised by the viscous length, $v / u_{\tau}$, where $v$ is the kinematic viscosity at the wall shear rate). It is, however, within the $95 \%$ confidence interval of the Virk profile (Graham 2014). In fact, it is well known (White, Dubief \& Klewicki 2012; Elbing et al. 2013) that data nominally following Virk's MDR asymptote are not truly logarithmic. Thus the MDR equation should be viewed as an idealisation that is helpful in highlighting that there exists a parameter regime where the velocity profile is only weakly sensitive to polymer and flow properties. As \%DR reduces due to polymer degradation, a decrease in the values of $U^{+}$can be observed, the velocity profiles smoothly interpolating between the MDR line and von Kármán log law for Newtonian fluids up to some $y^{+}$before becoming roughly parallel to the Newtonian log law (although this simple view is known to fail at high drag reduction where the log-law slope is no longer observed (Warholic, Massah \& Hanratty 1999)). A similar trend can be observed in the rectangular and square channels. 
Turbulent drag reduction by polymer additives in parallel-shear flows

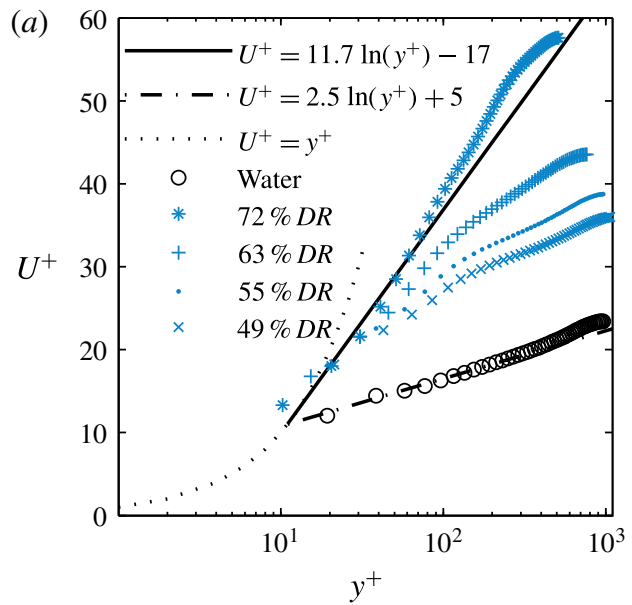

(b)

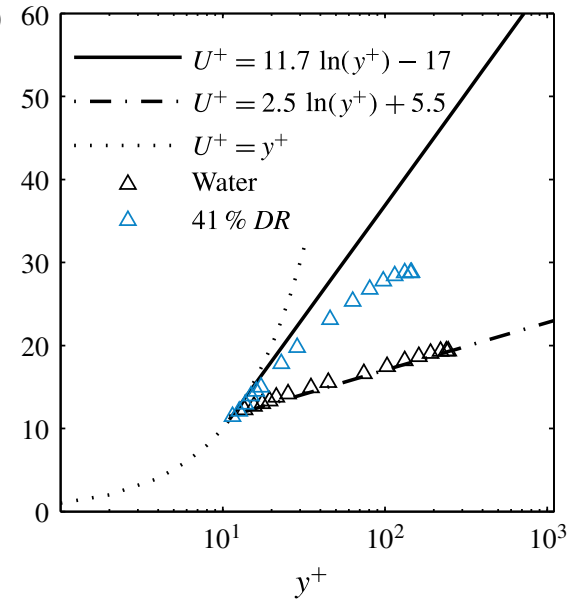

(c) 60

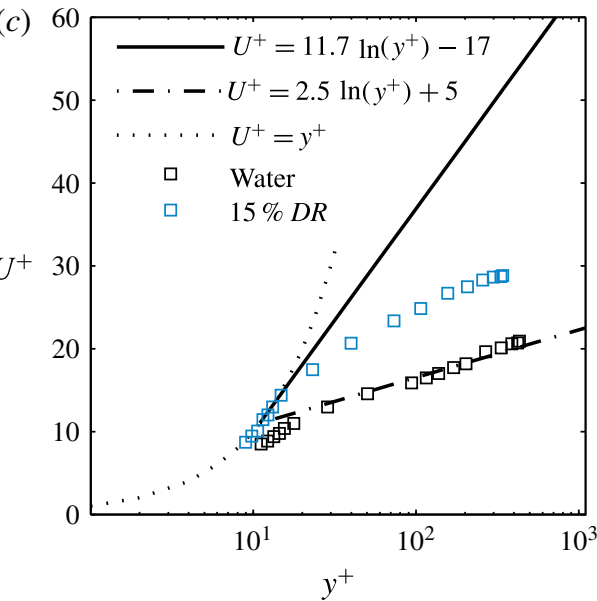

FIGURE 3. Time-averaged streamwise velocity profiles for 250 p.p.m. PAA solutions at various levels of drag reduction in three parallel-shear flows. (a) Azimuthally averaged data obtained using SPIV in the cylindrical pipe, (b) LDV data from the rectangular channel and (c) LDV profiles along the wall bisector of the square duct.

\section{Universal relationship between drag reduction and fluid elasticity}

The Fanning friction factors $(f)$ computed from pressure-drop measurements are shown in figure 4 . The measurements have been taken at different pumping times and mass flow rates and cover a wide range of \%DR. $f$ is plotted against the Reynolds number, $R e=\rho U_{b} h / \mu$, where $\rho, U_{b}, h$ and $\mu$ represent the density, bulk velocity, duct half-height (or radius in the case of the pipe) and apparent viscosity at the wall shear rate at any given $t$, respectively. The ranges of Reynolds number studied varied by roughly an order of magnitude in each geometry: $10^{3}-10^{4}$ for the channel, $4 \times 10^{3}-2 \times$ $10^{4}$ for the square duct and $5 \times 10^{3}-5 \times 10^{4}$ for the pipe. The approximate mean wall shear rate, estimated from shear viscosity curves, is the shear rate corresponding to $\tau_{w}$ computed from pressure-drop measurements at time $t$. This procedure is possible because the rheometer provides the link between shear stress and shear rate through the measured shear viscosity. We note that the mean shear rate determined in this 

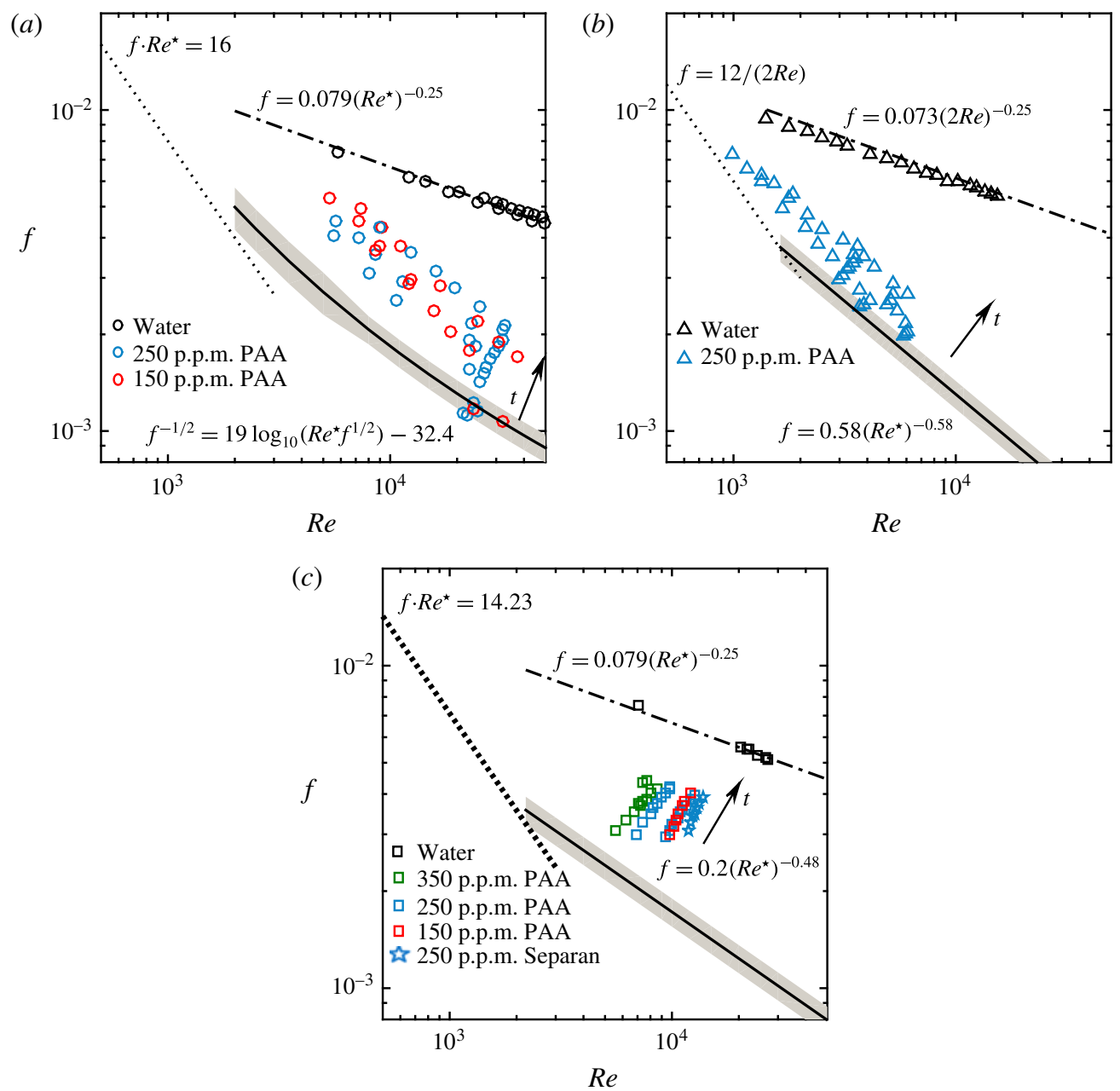

FIGURE 4. Fanning friction factors at different flow rates and pumping times for (a) cylindrical pipe, (b) rectangular channel and (c) square duct. Dotted lines $(\cdots)$ are the appropriate laminar flow equations for Fanning friction factor; dot-dashed lines (.---) are the correlations of Blasius (for pipe and square duct) and Dean (1978) (for rectangular channel); solid lines (- $)$ are the correlations of Virk (1975) (for pipe and rectangular channel) and Hartnett, Kwack \& Rao (1986) (for square duct) at MDR; the shaded regions represent $f= \pm 10 \%$ of MDR.

manner is merely an approximation for shear-thinning fluids and is exact only in the Newtonian limit (Housiadas \& Beris 2004). As expected, the polymer data lie between Virk's MDR and the correlation of Blasius or Dean (1978) for Newtonian turbulent flow, $f$ approaching the Newtonian values with increasing pumping time.

In their present form, figures 2 and 4 have very limited practical applicability in terms of predictive capability. It is therefore desirable to collapse the data for all three geometries onto a single curve. As a step towards this goal, we simply plot $\% D R$ against the Weissenberg number, $W i=\lambda_{c} \dot{\gamma}$, in figure 5(b). Quite remarkably, given the large range of Reynolds number, polymer concentration and different geometries, we observe excellent data collapse. The data are reasonably well fit $\left(R^{2}=0.82\right)$ by 

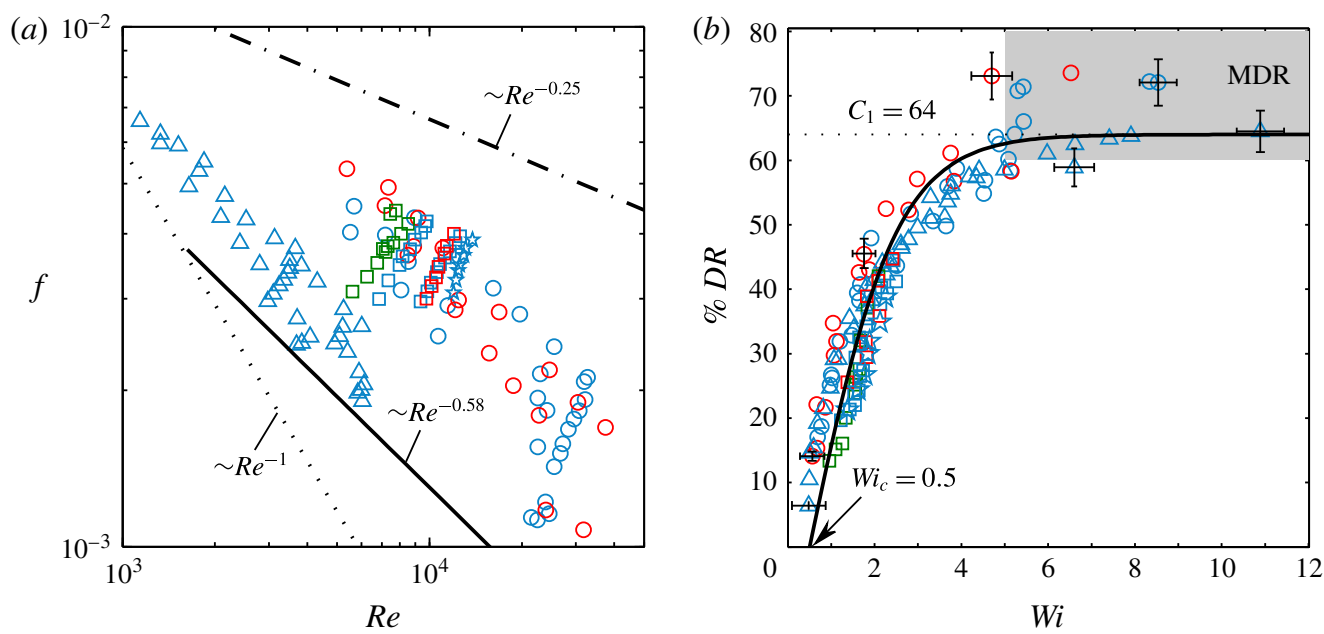

FiguRE 5. (a) Combined $f$-Re data for cylindrical pipe, rectangular channel and square duct (symbols and colours as in figure 4). (b) Variation of $\% D R$ with Weissenberg number. The solid black line represents the new correlation: $\% D R=2 C_{1}\left[1 /\left(1+\mathrm{e}^{W i_{c}-W i}\right)-W i_{c}\right]$.

the following equation:

$$
\% D R=2 C_{1}\left(\frac{1}{1+\mathrm{e}^{W_{c}-W i}}-W i_{c}\right)
$$

where $C_{1}=64$ is the approximate limiting value of $\% D R$ as $W i \rightarrow \infty$ and $W i_{c}$ is the critical Weissenberg number for the onset of drag reduction (set to be $\left.W i_{c}=0.5\right)$. Hence, we are able to relate the degree of drag reduction to a single measurable extensional rheological property of the polymer solutions independent of flow geometry, concentration, degradation and other experimental variables. The onset of $\% D R$ can be observed to take place at $W i \approx 0.5$, which, although in agreement with the theory of Lumley (1973), i.e. potentially related to the so-called 'coil-stretch' transition, may just be fortuitous because $W i$ here is based on the average wall shear rate rather than the fluctuating strain rates or the largest Lyapunov exponent (Stone \& Graham 2003). MDR is attained at $W i \gtrsim 5$, for which $\% D R$ becomes independent of Wi. We note a strong qualitative agreement between the universal form of our experimental data and results from direct numerical simulations (DNS) obtained from various models shown in Housiadas \& Beris (2013): however, the DNS data require an additional fitting parameter ('LDR'), which represents the asymptotic limit of large Weissenberg numbers and is not known a priori. In contrast, equation (5.1) enables the degree of drag reduction at a given flow rate in a given geometry to be estimated (using an iterative procedure) solely if the polymer shear rheology and relaxation time are known.

In observing a working functional dependence of $\% D R$ on $W i$ alone, the dependence on the ratio $\beta$ of solvent to total viscosity, on inertia (i.e. Reynolds number) and on other viscometric functions, e.g. first or second normal-stress differences, is neglected. All of the concentrations are quite similar, all being within the semidilute regime, so the effect of $\beta$ must contribute to the spread of the data around the fit. We note that the variation in the data is larger than the experimental uncertainty associated with the 


\section{B. E. Owolabi, D. J. C. Dennis and R. J. Poole}

data, which we estimate to be $\pm 3-3.5 \%$ as highlighted by the representative error bars shown in figure $5(b)$.

In the MDR limit it is also known that there remains some weak $R e$ dependence, as $\% D R$ scales roughly as $R e^{0.1}$ in this limit, and, as we have already discussed, there is significant spread in the literature for data nominally at MDR (Graham 2014), as is highlighted by the grey region in figure $5(b)$. Given the quality of the data collapse illustrated in figure $5(b)$, both $\beta$ and $R e$ seem to be second-order effects, at least for the concentrations (of the order of $\left.c^{*}\right)$ and range of $\operatorname{Re}\left(\approx 10^{3}-5 \times 10^{4}\right)$ studied for this particular flexible polymer. Although our data, and the correlation proposed in (5.1), suggest that no critical polymer concentration for drag-reduction onset is required (provided the mean wall shear rate is sufficient to make $W i \gtrsim 0.5$ ) we stress that the range of $\beta$ probed here $(\approx 0.1)$ means that the polymeric viscosity (and hence polymeric stress) is never negligible. In the limit where the solvent contribution to the stress dominates (i.e. $\beta \rightarrow 1$ ), simulations suggest that a critical concentration is required for onset regardless of shear rate (Lee \& Akhavan 2009).

We note also no dependence on the Trouton ratio $\operatorname{Tr}$ (i.e. the ratio of extensional viscosity to shear viscosity), but in our experiment we cannot independently adjust $\operatorname{Tr}$ and $\lambda_{c}$ because both are related to the length of the polymer molecule. Finally the data collected in Graham (2014) for different geometries - boundary layer, pipe and channel flow - also exhibit a similar spread, nominally at MDR, suggesting that some of the spread here could also be related to the use of different geometries and the simplistic estimate of the mean wall shear rate. In particular the square duct, and to a lesser extent the channel, exhibits a mean wall shear rate that varies around its periphery, whereas the pipe is axisymmetric and therefore spatially constant.

Finally, it is recommended that a similar procedure be applied to other types of flexible polymers to test the universality of the observed correlation between $\% D R$ and Wi. An attempt to obtain data for polyethylene oxide in our rigs was not successful, because the polymer degrades much more quickly, resulting in large variation in rheological properties within each rig at the same pumping times. Unfortunately such inhomogeneity of degradation precluded the same approach as was possible for the polyacrylamides.

\section{Conclusion}

In this study, turbulent drag reduction with polyacrylamide, a flexible polymer, has been investigated in a cylindrical pipe, a rectangular channel and a square duct. The polymer solutions were subjected to various levels of degradation by recirculating through the experimental rigs, and their shear and extensional rheological properties were closely monitored. Polymer relaxation times were observed to scale roughly as $t^{-0.5}$. Profiles of streamwise velocity $\left(U^{+}\right)$at various levels of drag reduction indicated a thickening of the buffer layer as observed in previous experimental studies, the buffer layer extending across the entire cross-section at MDR. A plot of $\% D R$ against Weissenberg number was found to collapse the data, with the onset of $\mathrm{DR}$ occurring at $W i \approx 0.5$ and $\mathrm{MDR}$ at $W i \gtrsim 5$, thus allowing for an a priori quantitative prediction of drag reduction from a knowledge of polymer relaxation time, flow rate and geometric length scale (using either the Newtonian pressure drop combined with rheology data, or the average shear rate as an initial guess to determine $W i$, combined with an iterative procedure). 
Turbulent drag reduction by polymer additives in parallel-shear flows

\section{Acknowledgements}

R.J.P. would like to thank the Engineering and Physical Sciences Research Council (EPSRC) for the award of a Fellowship under grant EP/M025187/1. We would also like to thank Professor M. Graham for helpful discussions and the anonymous referees for bringing a number of useful references to our attention.

\section{References}

Bazilevsky, A. V., Entov, V. M. \& Rozhkov, A. N. 1990 Liquid filament microrheometer and some of its applications. In Third European Rheology Conference and Golden Jubilee Meeting of the British Society of Rheology, pp. 41-43. Springer.

Clasen, C., Plog, J. P., Kulicke, W.-M., Owens, M., Macosko, C., Scriven, L. E., Verani, M. \& MCKinley, G. H. 2006 How dilute are dilute solutions in extensional flows? J. Rheol. 50 (6), 849-881.

DARBY, R. \& CHANG, H. F. D. 1984 Generalized correlation for friction loss in drag reducing polymer solutions. AIChE J. 30 (2), 274-280.

DEAN, R. B. 1978 Reynolds number dependence of skin friction and other bulk flow variables in two-dimensional rectangular duct flow. Trans. ASME J. Fluids Engng 100 (2), 215-223.

Den Toonder, J. M. J., DraAd, A. A., Kuiken, G. D. C. \& Nieuwstadt, F. T. M. 1995 Degradation effects of dilute polymer solutions on turbulent drag reduction in pipe flows. Appl. Sci. Res. 55 (1), 63-82.

Dennis, D. J. C. \& Sogaro, F. M. 2014 Distinct organizational states of fully developed turbulent pipe flow. Phys. Rev. Lett. 113 (23), 234501.

Dontula, P., Pasquali, M., Scriven, L. E. \& Macosko, C. C. W. 1997 Can extensional viscosity be measured with opposed-nozzle devices? Rheol. Acta 36 (4), 429-448.

Elbing, B. R., Perlin, M., Dowling, D. R. \& Ceccio, S. L. 2013 Modification of the mean near-wall velocity profile of a high-Reynolds number turbulent boundary layer with the injection of drag-reducing polymer solutions. Phys. Fluids 25 (8), 085103.

Escudier, M. P., Nickson, A. K. \& Poole, R. J. 2009 Turbulent flow of viscoelastic shearthinning liquids through a rectangular duct: quantification of turbulence anisotropy. J. NonNewtonian Fluid Mech. 160 (1), 2-10.

Escudier, M. P., Presti, F. \& SMith, S. 1999 Drag reduction in the turbulent pipe flow of polymers. J. Non-Newtonian Fluid Mech. 81 (3), 197-213.

GRAhAM, M. D. 2004 Drag reduction in turbulent flow of polymer solutions. Rheol. Rev. 2, $143-170$.

GRAHAM, M. D. 2014 Drag reduction and the dynamics of turbulence in simple and complex fluids. Phys. Fluids 26 (10), 101301.

Hartnett, J. P., KWACK, E. Y. \& RAO, B. K. 1986 Hydrodynamic behavior of non-Newtonian fluids in a square duct. J. Rheol. 30 (4), S45-S59.

HousiadAs, K. D. \& BERIS, A. N. 2003 Polymer-induced drag reduction: effects of the variations in elasticity and inertia in turbulent viscoelastic channel flow. Phys. Fluids 15 (8), 2369-2384.

HousiadAs, K. D. \& BERIS, A. N. 2004 Characteristic scales and drag reduction evaluation in turbulent channel flow of nonconstant viscosity viscoelastic fluids. Phys. Fluids 16 (5), 1581-1586.

HousiadAs, K. D. \& Beris, A. N. 2013 On the skin friction coefficient in viscoelastic wall-bounded flows. Intl J. Heat Fluid Flow 42, 49-67.

JAMES, D. F. \& YogACHANDRAN, N. 2006 Filament-breaking length-a measure of elasticity in extension. Rheol. Acta 46 (2), 161-170.

LEE, D.-H. \& AKHAVAN, R. 2009 Scaling of polymer drag reduction with polymer and flow parameters in turbulent channel flow. In Advances in Turbulence XII, pp. 359-362. Springer.

Lindner, A., VERMAnt, J. \& BOnN, D. 2003 How to obtain the elongational viscosity of dilute polymer solutions? Physica A 319, 125-133.

Lumley, J. L. 1973 Drag reduction in turbulent flow by polymer additives. J. Polym. Sci. Macromol. Rev. 7, 263-290. 


\section{B. E. Owolabi, D. J. C. Dennis and R. J. Poole}

Metzner, A. B. \& PARK, M. G. 1964 Turbulent flow characteristics of viscoelastic fluids. J. Fluid Mech. 20 (02), 291-303.

Owolabi, B. E., Poole, R. J. \& Dennis, D. J. C. 2016 Experiments on low-Reynolds-number turbulent flow through a square duct. J. Fluid Mech. 798, 398-410.

Procaccia, I., Lvov, V. S. \& BenzI, R. 2008 Colloquium: theory of drag reduction by polymers in wall-bounded turbulence. Rev. Mod. Phys. 80 (1), 225-247.

Purnode, B. \& Crochet, M. J. 1996 Flows of polymer solutions through contractions. Part 1: flows of polyacrylamide solutions through planar contractions. J. Non-Newtonian Fluid Mech 65 (2-3), 269-289.

Rodd, L. E., Scott, T. P., Cooper-White, J. J. \& McKinley, G. H. 2005 Capillary break-up rheometry of low-viscosity elastic fluids. Appl. Rheol. 15, 12-27.

Stone, P. A. \& Graham, M. D. 2003 Polymer dynamics in a model of the turbulent buffer layer. Phys. Fluids 15 (5), 1247-1256.

Sureshinumar, R., Beris, A. N. \& Handler, R. A. 1997 Direct numerical simulation of the turbulent channel flow of a polymer solution. Phys. Fluids 9 (3), 743-755.

Toms, B. A. 1948 Some observations on the flow of linear polymer solutions through straight tubes at large Reynolds numbers. In Proceedings of the 1st International Congress on Rheology, vol. 2, pp. 135-141. North-Holland.

VIRK, P. S. 1975 Drag reduction fundamentals. AIChE J. 21 (4), 625-656.

Warholic, M. D., Massah, H. \& Hanratty, T. J. 1999 Influence of drag-reducing polymers on turbulence: effects of Reynolds number, concentration and mixing. Exp. Fluids 27 (5), 461-472.

Whalley, R. D., Park, J. S., Kushwaha, A., Dennis, D. J. C., Graham, M. D. \& Poole, R. J. 2017 Low-drag events in transitional wall-bounded turbulence. Phys. Rev. Fluids 2, 034602.

White, C. M., Dubief, Y. \& Klewicki, J. 2012 Re-examining the logarithmic dependence of the mean velocity distribution in polymer drag reduced wall-bounded flow. Phys. Fluids 24 (2), 021701.

White, C. M. \& Mungal, M. G. 2008 Mechanics and prediction of turbulent drag reduction with polymer additives. Annu. Rev. Fluid Mech. 40, 235-256.

XI, L. \& GRAHAM, M. D. 2010 Turbulent drag reduction and multistage transitions in viscoelastic minimal flow units. J. Fluid Mech. 647, 421-452.

Zell, A., Gier, S., RAfAi, S. \& WAGNer, C. 2010 Is there a relation between the relaxation time measured in CaBER experiments and the first normal stress coefficient? J. Non-Newtonian Fluid Mech. 165 (19), 1265-1274. 